\title{
Viewpoint
}

\section{Does rheumatoid arthritis have a clinicopathological spectrum similar to that of leprosy?}

The diagnosis of rheumatoid arthritis is based on criteria which have been defined for epidemiological use. In clinical practice one frequently encounters problems of diagnosis, and one awaits the development of additional features before a firm diagnosis can be made. Furthermore, as individual patients are observed over months or years, changes in disease manifestations are encountered, nodules may come and go, vasculitis may appear in crops, erosions may develop with explosive rapidity or they may heal.

These events, which unpredictably happen during the many years of this chronic disease, illustrate a fundamental truth about rheumatoid arthritis: it is a dynamic, evolving condition so that clinically and immunopathologically an individual may be entirely different at each visit. The dynamic, changing nature of rheumatoid disease is best exemplified by the fate of extra-articular manifestations-cardiac, pulmonary, and vasculitic. ${ }^{1-4}$ This obvious truth tends to be forgotten in the hurly-burly of clinical practice. Forgetfulness is reinforced by the lessons learned from some experimental animal models which, following an explosive initiation phase, have a chronic course characterised by a decrescendo healing phase.

\section{CLINICAL SPECTRUM}

The term 'clinical spectrum' of rheumatoid disease implies that its various manifestations (articular and extra-articular) are components of the same disease. Two extremes of this spectrum will therefore be examined. At one end is the patient who presents with rheumatoid nodules, little or no synovitis, a high titre of rheumatoid factor, and no erosions on radiology of the joints. The patient may continue in this state for months or even years before joint pathology with radiological erosions finally supervenes. At the opposite end of this spectrum is the patient with synovitis of varying degree, who has radiological evidence of joint damage but who is seronegative for rheumatoid factor. It is the experience of most rheumatologists that such a patient, if he or she remains in this phase, will have a good prognosis, with arrest of disease and even healing of erosions.

In between are those patients who have erosions, extra-articular manifestations, and high titres of rheumatoid factor. However, we have already noted that these patients are not in a fixed state. Rather they are in an unstable dynamic state especially noticeable in the variation of the number, severity, and course of extra-articular complications. Even the titre of rheumatoid factor itself may change with time independently of effective therapy. So it is obvious that, so far as these manifestations of rheumatoid disease are concerned, patients may fluctuate from one end of the spectrum to the other.

A clinical spectrum, therefore, in the expression of extra-articular features of rheumatoid disease seems unquestionable. But, it may be objected, the majority of patients are seropositive and have erosive disease, so what is the relevance of proposing a clinicalo spectrum whose only usefulness is the trivial one of allowing us to 'classify' a handful of patients? This objection is invalid for 2 reasons. First, clinical $\stackrel{+}{?}$ diagnostic criteria have been developed for epidemiological purposes, so that in fact the majority of patients with RA may not have the features of the disease which we see in the clinic. Indeed there is evidence from radiological surveys of large populations that healed erosive disease is probably as common as clinically apparent disease. ${ }^{5} \mathrm{Or}$, to put it another way, the illness seen in clinics is highly selected and does not represent the real disease as it is present in the population. Secondly, although most extra-articular manifestations may come and go (nodules, vasculitis), erosions may take years to heal, so that their presence at any one moment does not necessarily mean that the processes which give rise to them are active at that time. It also follows that recurrent activation of these processes will inevitably lead to an accumulating load of joint damage. Under these circumstances it is not surprising that, so far as joint erosions are concerned, one infrequently observes dynamic change in joint destruction.

IMMUNOPATHOLOGICAL SPECTRUM

The definition of a clinical spectrum of rheumatoid disease demands the analogous definition of an immunopathological spectrum, since the former will be determined by the latter. In defining this disease spectrum leprosy will be used as the model. ${ }^{6}$ In 
leprosy the extreme ends of the spectrum are the lepromatous and the tuberculoid. The former is characterised by the predominance of humoral immunity (hypergammaglobulinaemia, autoantibodies, circulating immune complexes) and the absence of cellular immunity (negative lepromin reaction), while the latter is characterised by the predominance of cellular immunity (positive lepromin reaction) and the absence of humoral immunity. There are, of course, intermediate or borderline forms of the disease in which patients have features of the 2 extremes in varying proportions. Death occurs predominantly in the lepromatous stage from the complications of immune-complex-mediated disease, while during the tuberculoid stage there is activation of cell-mediated immunity with destruction of tissues. If a patient has any chance of 'curing' his disease, he can do this only during the tuberculoid phase. Indeed this is the usual outcome of the infection as attested to by the many persons who have successfully survived infection whose only sign is a positive lepromin reaction. Finally, it is most important to remember that a patient may fluctuate from one part of the spectrum to another: leprosy is a dynamic disease.

How does rheumatoid disease fit into this concept ${ }^{8}$ First, evidence has been presented which suggests that RA is a dynamic rather than a static state. Secondly, it has been indicated that extreme and intermediate forms of the disease do indeed exist on clinical grounds. Thirdly inflammatory and extra-articular features are though to be mediated by humoral mechanisms (deposition of immune complexes and activation of the complement system) while joint erosions are thought to be the result of cell-mediated immune mechanisms involving lymphocytes. ${ }^{8}$ Fourthly, death occurs predominantly during immune complex phases (presence of extra-articular manifestations) of the disease. ${ }^{9}$ Fifthly, seronegative disease is the most likely to 'burn itself out' early and with only minimal erosive damage to joints. Finally, if we had the rheumatoid equivalent of the lepromin test we might be able to show that a large number of people have indeed successfully met the aetiological agent of RA, the only evidence of the event being a positive 'rheumatin' test.

It is therefore proposed that rheumatoid disease is a dynamic disease with a clinical and immunopathological spectrum, namely: (1) lepromatous rheumatoid: immune complex disease with extraarticular complications; (2) tuberculoid rheumatoid: cell-mediated immune disease with joint destruction. There are, of course, intermediate or borderline phases with features of both extremes being present, and patients may oscillate from one part of the spectrum to another.

Some of the studies referred to were financed by grants from the Arthritis and Rheumatism Council for Great Britain.

\section{G. S. PANAYI \\ Professor of Rheumatology, Guy's Hospital Medical School, London SE1 9RT}

\section{References}

1 Eberl R, Rosenthal M. Organic Manifestations and Complications in RA. Stuttgart: Schattauer, 1976.

2 Iveson J M I, Pomerance A. Cardiac involvement in rheumatoid disease. Clin Rheum Dis 1977; 3: 467-500.

3 Turner-Warwick M, Evans R C. Pulmonary manifestations of rheumatoid disease. Clin Rheum Dis 1977; 3: 549-64.

4 Zvaifler $\mathrm{N}$ J. The immunopathology of joint inflammation in rheumatoid arthritis. Adv Immunol 1973; 16: 265-36.

5 Kellgren J H. Epidemiology of rheumatoid arthritis. In: Duthie J J R, Alexander W $\mathbf{R} \mathbf{M}$, eds. Rheumatic Diseases. Edinburgh University Press, 1968: 7-18.

6 Myrvang B, Godal T, Ridley D S, Froland S S, Song Y K. Immune responsiveness to Mycobacterium leprae and other mycobacterial antigens throughout the clinical and histopathological spectrum of leprosy. Clin Exp Immunol 1973; 14: 541-53.

7 Ridley D S, Jopling W H. Classification of leprosy according to immunity: a five group system. Int $J$ Lepr 1966; 34: 255-61.

8 Panayi G S. Pathogenesis of rheumatoid arthritis. Eur $J$ Rheumatol Inflamm 1979; 2: 265-71.

9 Gordon D A, Stein J L, Broder I, The extra-articular manifestations of rheumatoid arthritis. A systemic analysis of 127 cases. Am J Med 1973; 54: 445-52. 